\title{
MIXED PROBLEM WITH INTEGRAL BOUNDARY CONDITION FOR A HIGH ORDER MIXED TYPE PARTIAL DIFFERENTIAL EQUATION
}

\author{
M. DENCHE and A.L. MARHOUNE \\ Université Mentouri Constantine \\ Institut de Mathematiques \\ 25000 Constantine, Algeria
}

(Received April, 2000; Revised March, 2002)

\begin{abstract}
In this paper, we study a mixed problem with integral boundary conditions for a high order partial differential equation of mixed type. We prove the existence and uniqueness of the solution. The proof is based on energy inequality, and on the density of the range of the operator generated by the considered problem.

Key words: Integral Boundary Condition , Energy Inequalities, Equation of Mixed Type, Sobolev Spaces.

AMS subject classifications: $35 \mathrm{~B} 45,35 \mathrm{G} 10,35 \mathrm{M} 10$.
\end{abstract}

\section{Introduction}

In the rectangle $Q=(0,1) \times(0, T)$, we consider the equation

$$
\mathcal{L} u=\frac{\partial^{2} u}{\partial t^{2}}+(-1)^{\alpha} \frac{\partial^{\alpha}}{\partial x^{\alpha}}\left(a(x, t) \frac{\partial^{\alpha+1} u}{\partial x^{\alpha} \partial t}\right)=f(x, t),
$$

where $a(x, t)$ is bounded for $0<a_{0}<a(x, t) \leq a_{1}$, and has bounded partial derivatives such that $0<a_{2} \leq \frac{\partial a(x, t)}{\partial t} \leq a_{3}$ and $\left|\frac{\partial^{\mathrm{i}} a(x, t)}{\partial x i}\right| \leq b_{i}, i=\overline{1, \alpha}$, for $(x, t) \in \bar{Q}$.

To equation (1) we add the initial conditions

$$
l_{1} u=(x, 0)=\varphi(x), l_{2} u=\frac{\partial u}{\partial t}(x, 0)=\psi(x) \quad x \in(0,1)
$$

the boundary conditions

$$
\begin{aligned}
& \frac{\partial^{i}}{\partial x^{i}} u(0, t)=0, \text { for } 0 \leq i \leq \alpha-1, t \in(0, T), \\
& \frac{\partial^{i}}{\partial x^{i}} u(1, t)=0, \text { for } 0 \leq i \leq \alpha-2, t \in(0, T),
\end{aligned}
$$

and integral condition 


$$
\int_{0}^{1} u(\xi, t) d \xi=0, \quad t \in(0, T)
$$

where $\varphi$ and $\psi$ are known functions which satisfy the compatibility conditions given in equations (3)-(5).

Integral boundary conditions for evolution problems have various applications in chemical engineering, thermoelasticity, underground water flow and population dynamics; see for example Choi and Chan [6], Ewing and Lin [7], Shi [12], and Shi and Shillor [13]. Boundary value problems for parabolic equations with integral boundary conditions are investigated by Batten [1], Bouziani and Benouar [2], Cannon [3, 4], Cannon, Perez Esteva and Van der Hoeck [5], Ionkin [8], Kamynin [9], Kartynnik [10], Shi [12], Yurchuk [14] and many references therein. A method was developed in [2], [10], and [14] based on energy inequalities. In this paper, our objective is to extend this method to high order partial differential equations of mixed type.

\section{Preliminaries}

In this paper, we prove existence and uniqueness of a strong solution of the problem stated in equations (1)-(5). For this, we consider the problem in equations (1)-(5) as a solution of the operator equation

$$
L u=\mathcal{F},
$$

where $L=\left(\mathcal{L}, l_{1}, l_{2}\right)$, with domain of definition $D(L)$ consisting of functions $u \in W_{2}^{2 \alpha, 2}(Q)$, such that, $\frac{\partial^{i+1} u}{\partial x^{i} \partial t} \frac{\partial^{i}}{\partial x^{i}}\left(\frac{\partial^{\alpha+1} u}{\partial x^{\alpha} \partial t}\right) \in L_{2}(Q), i=\overline{1, \alpha}$ and, $u$ satisfies conditions in problems (3)-(5); the operator $L$ is considered from $E$ to $F$, where $E$ is the Banach space consisting of all functions $u \in L_{2}(Q)$, satisfying equations (3)-(5), with the finite norm

$$
\begin{gathered}
\|u\|_{E}^{2}=\int_{Q}(1-x)\left[\left|\frac{\partial^{2} u}{\partial t^{2}}\right|^{2}+\left|\frac{\partial^{\alpha}}{\partial^{\alpha}}\left(a \frac{\partial^{\alpha+1} u}{\partial x^{\alpha} \partial t}\right)\right|^{2}\right] d x d t \\
+\sum_{i=1}^{\alpha-1} \int_{Q} x^{2 i}(1-x)^{2 i+1}\left|\frac{\partial^{i}}{\partial x^{i}}\left(a \frac{\partial^{\alpha+1} u}{\partial x^{\alpha} \partial t}\right)\right|^{2} d x d t \\
+\sup _{0 \leq t \leq T} \int_{0}^{1}(1-x)\left[\sum_{i=0}^{a}\left|\frac{\partial^{i} u}{\partial x^{i}}\right|^{2}+\left|\frac{\partial^{\alpha+1} u}{\partial x^{\alpha} \partial t}\right|^{2}+\left|\frac{\partial u}{\partial t}\right|^{2}\right] d x,
\end{gathered}
$$

and $F$ is the Hilbert space of vector-valued functions $\mathcal{F}=(f, \varphi, \psi)$ obtained by completion of the space $L_{2}(Q) \times W_{2}^{2 \alpha, 2}(0,1) \times W_{2}^{2 \alpha, 2}(0,1)$ with respect to the norm

$$
\|\mathcal{F}\|_{F}^{2}=\int_{Q}(1-x)^{\nu}|f|^{2} d x d t+\int_{0}^{1}(1-x)\left[\sum_{i=0}^{a}\left|\frac{\partial^{i} \varphi}{\partial x^{i}}\right|^{2}+\left|\frac{\partial^{\alpha} \psi}{\partial x^{\alpha}}\right|^{2}+|\psi|^{2}\right] d x
$$

where $\nu$ is an arbitrary number such that $0<\nu<1$.

We then establish an energy inequality: 


$$
\|u\|_{E} \leq C_{1}\|L u\|_{F}
$$

and we show that the operator $L$ has the closure $\bar{L}$.

Definition 1: A solution of the operator equation $\bar{L} u=\mathcal{F}$ is called a strong solution of the problem in equations (1)-(5).

Inequality (9) can be extended to $u \in D(\bar{L})$, i.e.,

$$
\|u\|_{E} \leq C_{1}\|\bar{L} u\|_{F}, \quad \forall u \in D(\bar{L}) .
$$

From this inequality, we obtain the uniqueness of a strong solution if it exists, and the equality of sets $R(\bar{L})$ and $\overline{R(L)}$. Thus, to prove the existence of a strong solution of the problem in equations (1)-(5) for any $\mathcal{F} \in F$, it remains to prove that the set $R(L)$ is dense in $F$.

Lemma 1: For any function $u \in E$ satisfying the condition (2), for $c \geq 8$

$$
\begin{gathered}
\int_{0}^{1} \exp (-c \tau)(1-x)\left|\frac{\partial u}{\partial t}(x, \tau)\right|^{2} d x \leq \int_{0}^{1}(1-x)|\psi|^{2} d x \\
+\frac{1}{8} \int_{0}^{\tau} \int_{0}^{1} \exp (-c t)(1-x)\left|\frac{\partial^{2} u}{\partial t^{2}}\right|^{2} d x d t .
\end{gathered}
$$

Proof: Starting from $\int_{0}^{\tau} \int_{0}^{1} \exp (-c t)(1-x) \frac{\partial^{2} u}{\partial t^{2}} \frac{\partial \bar{u}}{\partial t} d x d t$ and integrating by parts, we obtain equation (10).

Remark 1: We note the above lemma holds for weaker conditions on $u$.

Lemma 2: For $u \in E$,

$$
\int_{0}^{1}(1-x)\left|\frac{\partial^{\alpha} u}{\partial x^{\alpha-1} \partial t}\right|^{2} d x \leq \int_{0}^{1}(1-x)\left|\frac{\partial^{\alpha+1} u}{\partial x^{\alpha} \partial t}\right|^{2} d x .
$$

Proof: Starting from $\int_{0}^{1}(1-x) \frac{\partial^{\alpha+1} u}{\partial x^{\alpha} \partial t} \frac{\partial^{\alpha} \bar{u}}{\partial x^{\alpha-1} \partial t} d x$ and using elementary inequalities yields (11).

Lemma 3: For $u \in E$ satisfying the condition in equation (2),

$$
\begin{gathered}
\sum_{i=0}^{\alpha} \int_{0}^{1} \exp (-c \tau)(1-x)\left|\frac{\partial^{i} u(x, \tau)}{\partial x^{i}}\right|^{2} d x \leq \sum_{i=0}^{\alpha} \int_{0}^{1}(1-x)\left|\frac{\partial^{i} \varphi}{\partial x^{i}}\right|^{2} d x \\
+(\alpha+1) \int_{0}^{\tau} \int_{0}^{1} \exp (-c t)(1-x)\left|\frac{\partial^{\alpha+1} u}{\partial x^{\alpha} \partial t}\right|^{2} d x d t
\end{gathered}
$$

Proof: Integrating the expression $\int_{0}^{\tau} \exp (-c t) \frac{\partial^{i+1} u}{\partial x^{i} \partial t} \frac{\partial^{i} u}{\partial x^{i}} d t$ by parts for $i=\overline{0, \alpha}$, using elementary inequalities and Lemma 2, we obtain expression (12).

\section{An Energy Inequality and Its Consequences}

Theorem 1: For any function $u \in D(L)$ we have the inequality

$$
\|u\|_{E} \leq C_{1}\|L u\|_{F}
$$


where constant $C_{1}=\frac{\exp (c T) \max \left(\left(4 \alpha^{2} /(1-\nu)^{2}\right)+\frac{9}{8}, a_{1}\right)}{\min \left(\frac{1}{64}, a_{0}\right)}$, with the constant c satisfying

$$
c \geq 8 \text { and } c a_{0}-(\alpha+1) \geq a_{3}+\frac{a_{1}}{16}\left(3 \beta_{1}-6\right) .
$$

Proof: Denote

$$
M u=(1-x) \frac{\partial^{2} u}{\partial t^{2}}+\alpha J \frac{\partial^{2} u}{\partial t^{2}} \text { where } J u=\int_{0}^{x} u(\xi, t) d \xi .
$$

We consider the quadratic formula

$$
\operatorname{Re} \int_{0}^{\tau} \int_{0}^{1} \exp (-c t) \mathcal{L} u \overline{M u} d x d t
$$

with the constant $c$ satisfying condition (14); obtained by multiplying equation (1) by $\exp (-c t) \overline{M u}$; integrating over $Q^{\tau}$, where $Q^{\tau}=(0,1) \times(0, \tau)$, with $0 \leq \tau \leq T$; and by taking the real part. Integrating by parts $\alpha$ times in formula (15) with the use of boundary conditions in equations (3), (4), and (5), we obtain

$$
\begin{gathered}
\operatorname{Re} \int_{0}^{\tau} \int_{0}^{1} \exp (-c t) \mathcal{L} u \overline{M u} d x d t=\int_{0}^{\tau} \int_{0}^{1} \exp (-c t)(1-x)\left|\frac{\partial^{2} u}{\partial t^{2}}\right|^{2} d x d t \\
+\operatorname{Re} \int_{0}^{\tau} \int_{0}^{1} \exp (-c t)(1-x) a \frac{\partial^{\alpha+1} u}{\partial x^{\alpha} \partial t} \frac{\partial^{\alpha+2} \bar{u}}{\partial x^{\alpha} \partial t^{2}} d x d t
\end{gathered}
$$

By substituting the expression of $M u$ in formula (15), using elementary inequalities and the inequality

yields:

$$
\int_{0}^{1} \frac{\left|J \frac{\partial^{2} u}{\partial t^{2}}\right|^{2}}{(1-x)^{\nu}} d x \leq \frac{4}{(1-\nu)^{2}} \int_{0}^{1}(1-x)\left|\frac{\partial^{2} u}{\partial t^{2}}\right|^{2} d x, \text { where } 0<\nu<1
$$

$$
\begin{gathered}
\operatorname{Re} \int_{0}^{\tau} \int_{0}^{1} \exp (-c t) \mathcal{L} u \overline{M u} d x d t \leq\left(\frac{4 \alpha^{2}}{(1-\nu)^{2}}+1\right) \int_{0}^{\tau} \int_{0}^{1} \exp (-c t)(1-x)^{\nu}|\mathcal{L} u|^{2} d x d t \\
+\frac{1}{2} \int_{0}^{\tau} \int_{0}^{1} \exp (-c t)(1-x)\left|\frac{\partial^{2} u}{\partial t^{2}}\right|^{2} d x d t
\end{gathered}
$$

From equation (1) we have:

$$
\frac{1}{2}\left|\frac{\partial^{\alpha}}{\partial x^{\alpha}}\left(a \frac{\partial^{\alpha+1} u}{\partial x^{\alpha} \partial t}\right)\right|^{2} \leq|\mathcal{L} u|^{2}+\left|\frac{\partial^{2} u}{\partial t^{2}}\right|^{2}
$$

Using elementary inequalities, we obtain

$$
\frac{1}{4} \sum_{i=1}^{\alpha-1} \int_{0}^{\tau} \int_{0}^{1} \exp (-c t) x^{2 i}(1-x)^{2 i+1}\left|\frac{\partial^{i}}{\partial x^{i}}\left(a \frac{\partial^{\alpha+1} u}{\partial x^{\alpha} \partial t}\right)\right|^{2} d x d t
$$




$$
\begin{gathered}
\leq \frac{1}{2} \int_{0}^{\tau} \int_{0}^{1} \exp (-c t)(1-x)\left|\frac{\partial^{\alpha}}{\partial x^{\alpha}}\left(a \frac{\partial^{\alpha+1} u}{\partial x^{\alpha} \partial t}\right)\right|^{2} d x d t \\
+\left(3 \beta_{1}-6\right) \int_{0}^{\tau} \int_{0}^{1} a \exp (-c t)(1-x)\left|\frac{\partial^{\alpha+1} u}{\partial x^{\alpha} \partial t}\right|^{2} d x d t,
\end{gathered}
$$

where $\beta_{1}>0$.

By integrating the last term on the right-hand side of expression (16) and combining the obtained results with Lemmas 1 and 3, and the inequalities in equations (17), (18), and (19), we get:

$$
\begin{gathered}
\left(\frac{4 \alpha^{2}}{(1-\nu)^{2}}+\frac{9}{8}\right) \int_{0}^{\tau} \int_{0}^{1} \exp (-c t)(1-x)^{\nu}|\mathcal{L} u|^{2} d x d t+a_{1} \int_{0}^{1}(1-x)\left|\frac{\partial^{\alpha} \psi}{\partial x^{\alpha}}\right|^{2} d x+\int_{0}^{1}(1-x)|\psi|^{2} d x \\
+\sum_{i=1}^{\alpha} \int_{0}^{1}(1-x)\left|\frac{\partial^{i} \varphi}{\partial x^{i}}\right|^{2} d x \geq \sum_{i=0}^{\alpha} \int_{0}^{1} \exp (-c \tau)(1-x)\left|\frac{\partial^{i} u(x, \tau)}{\partial x^{i}}\right|^{2} d x \\
+\frac{2}{8} \int_{0}^{\tau} \int_{0}^{1} \exp (-c t)(1-x)\left|\frac{\partial^{2} u}{\partial t^{2}}\right|^{2} d x d t \\
+a_{0} \int_{0}^{1} \exp (-c \tau)(1-x)\left|\frac{\partial^{\alpha+1} u(x, \tau)}{\partial x^{\alpha} \partial t}\right|^{2} d x+\frac{1}{32} \int_{0}^{\tau} \int_{0}^{1} \exp (-c t)(1-x)\left|\frac{\partial^{\alpha}}{\partial x^{\alpha}}\left(a \frac{\partial^{\alpha+1} u}{\partial x^{\alpha} \partial t}\right)\right|^{2} d x d t \\
+\frac{1}{64} \sum_{i=1}^{\alpha-1} \int_{0}^{\tau} \int_{0}^{1} \exp (-c t) x^{2 i}(1-x)^{2 i+1}\left|\frac{\partial^{i}}{\partial x^{i}}\left(a \frac{\partial^{\alpha+1} u}{\partial x^{\alpha} \partial t}\right)\right|^{2} d x d t \\
+\left.\left.\int_{0}^{1} \exp (-c \tau)(1-x)\right|^{2} \frac{\partial u(x, \tau)}{\partial t}\right|^{2} d x \\
+\left(c a_{0}-a_{3}-(\alpha+1)-a_{1}\left(\frac{3 \beta_{1}-6}{16}\right)\right) \int_{0}^{\tau} \int_{0}^{1} \exp (-c t)(1-x)\left|\frac{\partial^{\alpha+1} u}{\partial x^{\alpha} \partial t}\right|^{2} d x d t .
\end{gathered}
$$

Using elementary inequalities and condition (14), we obtain:

$$
\begin{gathered}
\left(\frac{4 \alpha^{2}}{(1-\nu)^{2}}+\frac{9}{8} \int_{Q}(1-x)^{\nu}|\mathcal{L} u|^{2} d x d t+a_{1} \int_{0}^{1}(1-x)\left|\frac{\partial^{\alpha} \psi}{\partial x^{\alpha}}\right|^{2} d x+\int_{0}^{1}(1-x)|\psi|^{2} d x\right. \\
+\sum_{i=0}^{\alpha} \int_{0}^{1}(1-x)\left|\frac{\partial^{i} \varphi}{\partial x^{i}}\right|^{2} d x \\
\geq \exp (-c T)\left[\sum_{i=0}^{\alpha} \int_{0}^{1}(1-x)\left|\frac{\partial^{i} u(x, \tau)}{\partial x^{i}}\right|^{2} d x+\frac{2}{8} \int_{0}^{\tau} \int_{0}^{1}(1-x)\left|\frac{\partial^{2} u}{\partial t^{2}}\right|^{2} d x d t\right. \\
+a_{0} \int_{0}^{1}(1-x)\left|\frac{\partial^{\alpha+1} u(x, \tau)}{\partial x^{\alpha} \partial t}\right|^{2} d x+\frac{1}{32} \int_{0}^{\tau} \int_{0}^{1}(1-x)\left|\frac{\partial^{\alpha}}{\partial x^{\alpha}}\left(a \frac{\partial^{\alpha+1} u}{\partial x^{\alpha} \partial t}\right)\right|^{2} d x d t
\end{gathered}
$$




$$
\left.+\frac{\mathbf{1}}{\mathbf{6 4}} \sum_{i=0}^{\alpha-1} \int_{0}^{\tau} \int_{0}^{1} x^{2 i}(1-x)^{2 i+1}\left|\frac{\partial^{i}}{\partial x^{i}}\left(a \frac{\partial^{\alpha+1} u}{\partial x^{\alpha} \partial t}\right)\right|^{2} d x d t+\int_{0}^{1}(1-x)\left|\frac{\partial u(x, \tau)}{\partial t}\right|^{2} d x\right] \text { • }
$$

As the left-hand side of equation (21) is independent of $\tau$, by replacing the right-hand side by its upper bound with respect to $\tau$ in the interval $[0, T]$, we obtain the desired inequality.

Lemma 4: The operator $L$ from $E$ to $F$ admits a closure.

Proof: Suppose that $\left(u_{n}\right) \in D(L)$ is a sequence such that

$$
u_{n} \rightarrow 0 \text { in } E,
$$

and

$$
L u_{n} \rightarrow \mathcal{F} \text { in } F
$$

We must show that $\mathcal{F}=\left(f, \varphi_{1}, \varphi_{2}\right)=0$. The fact that $\varphi_{i}=0 ; i=1,2$ results directly from the continuity of the trace operators $l_{i}$.

Introducing the operator

$$
\mathcal{L}_{0} v=\frac{\partial^{2} v}{\partial t^{2}}+(-1)^{\alpha+1} \frac{\partial^{\alpha+1}}{\partial x^{\alpha} \partial t}\left(a(x, t) \frac{\partial^{\alpha} v}{\partial x^{\alpha}}\right)
$$

defined on the domain $D\left(\mathcal{L}_{0}\right)$ of functions $v \in W_{2}^{2 \alpha, 2}(Q)$ verifying

$$
\left.v\right|_{t=T}=0,\left.\frac{\partial v}{\partial t}\right|_{t=T}=0,\left.\frac{\partial^{i} v}{\partial x i}\right|_{x=0}=0, i=\overline{0, \alpha-1},\left.\frac{\partial^{i} v}{\partial x^{i}}\right|_{x=1}=0, i=\overline{0, \alpha}
$$

we note that $D\left(\mathcal{L}_{0}\right)$ is dense in the Hilbert space obtained from the completion of $L_{2}(Q)$ with respect to the norm

$$
\|v\|^{2}=\int_{Q}(1-x)^{\nu}|v|^{2} d x d t
$$

Additionally, since

$$
\int_{Q}(1-x)^{\nu} f \bar{v} d x d t=\lim _{n \rightarrow \infty} \int_{Q} \mathcal{L} u_{n}(1-x)^{\nu} \bar{v} d x d t=\lim _{n \rightarrow \infty} \int_{Q} u_{n} \mathcal{L}_{0}\left((1-x)^{\nu} \bar{v}\right) d x d t=0
$$

this holds for every function $v \in D\left(\mathcal{L}_{0}\right)$, and yields $f=0$.

Theorem 1 is valid for strong solutions, i.e., we have the inequality

$$
\|u\|_{E} \leq C_{1}\|\bar{L} u\|_{F}, \quad \forall u \in D(\bar{L}),
$$

hence we obtain

Corollary 1: A strong solution of the problem in equations (1)-(5) is unique if it exists, and depends continuously on $\mathcal{F}=(f, \varphi, \psi) \in F$.

Corollary 2: The range $R(L)$ of the operator $\bar{L}$ is closed in $F$, and $R(\bar{L})=\overline{R(L)}$.

\section{Solvability of the Problem}


To prove the solvability of the problem in equations (1)-(5), it is sufficient to show that $R(L)$ is dense in $F$. The proof is based on the following lemma.

Lemma 5: Suppose that $\left|\frac{\partial^{i+1} a(x, t)}{\partial x^{i} \partial t}\right| \leq b_{i}, i=\overline{1, \alpha}$, is also bounded. Let $D_{0}(L)=\{u \in$ $\left.D(L): l_{1} u=0, l_{2} u=0\right\}$. If, for $u \in D_{0}(L)$ and some $\omega \in L_{2}(Q)$, we have

$$
\int_{Q}(1-x)^{\alpha+\nu+1} \mathcal{L} u \varpi d x d t=0
$$

where $\nu$ is an arbitrary number such that $0<\nu<1$, then $\omega=0$.

Proof: The equality (24) can be written as follows:

$$
-\int_{Q}(1-x)^{\alpha+\nu+1} \frac{\partial^{2} u}{\partial t^{2}} \varpi d x d t=(-1)^{\alpha} \int_{Q}(1-x)^{\alpha+\nu+1} \frac{\partial^{\alpha}}{\partial x^{\alpha}}\left(a \frac{\partial^{\alpha+1} u}{\partial x^{\alpha} \partial t}\right) \varpi d x d t .
$$

If we introduce the smoothing operators with respect to $t[14] J_{\epsilon}^{-1}=\left(I+\epsilon \frac{\partial}{\partial t}\right)^{-1}$ and $\left(J_{\epsilon}^{-1}\right)^{*}$, then these operators provide the solutions of the problems

$$
\begin{gathered}
\epsilon \frac{d g_{\epsilon}(t)}{d t}+g_{\epsilon}(t)=g(t), \\
\left.\mathrm{g}_{\epsilon}(t)\right|_{t=0}=0
\end{gathered}
$$

and

$$
\begin{gathered}
-\epsilon \frac{d g_{\epsilon}^{*}(t)}{d t}+g_{\epsilon}^{*}(t)=g(t), \\
\left.g_{\epsilon}^{*}(t)\right|_{t=T}=0,
\end{gathered}
$$

and also have the following properties: for any $g \in L_{2}(0, T)$, the functions $g_{\epsilon}=\left(J_{\epsilon}^{-1}\right) g$ and $g_{\epsilon}^{*}=\left(J_{\epsilon}^{-1}\right)^{*} g$ are in $W_{2}^{1}(0, T)$ such that $\left.g_{\epsilon}\right|_{t=0}=0$ and $\left.g_{\epsilon}^{*}\right|_{t=T}=0$. Moreover, $J_{\epsilon}^{-1}$ commutes with $\frac{\partial}{\partial t}$, so $\int_{0}^{T}\left|g_{\epsilon}-g\right|^{2} d t \rightarrow 0$ and $\int_{0}^{T}\left|g_{\epsilon}^{*}-g\right|^{2} d t \rightarrow 0$, for $\epsilon \rightarrow 0$.

Now, for given $\omega(x, t)$, we introduce the function

$$
v(x, t)=(1-x)^{\alpha-1} \int_{0}^{x}(1-\xi)^{\nu+1} \frac{\partial \omega(\xi, t)}{\partial \xi} d \xi-(\alpha+\nu+1)(1-x)^{\alpha-1} \int_{0}^{x}(1-\xi)^{\nu} \omega(\xi, t) d \xi
$$

Integrating by parts, we obtain

$$
(1-x) v+\alpha J v=(1-x)^{\alpha+\nu+1} \omega, \text { and } \int_{0}^{1} v(x, t) d x=0 .
$$

Then from equality (25), we have

$$
-\int_{Q} \frac{\partial^{2} u}{\partial t^{2}} N \bar{v} d x d t=\int_{Q} A(t) \frac{\partial u}{\partial t} \bar{v} d x d t
$$

where $N v=(1-x) v+\alpha J v$, and $A(t) u=(-1)^{\alpha} \frac{\partial^{\alpha}}{\partial x^{\alpha}}\left((1-x) a(x, t) \frac{\partial^{\alpha} u}{\partial x^{\alpha}}\right)$. Replacing $\frac{\partial u}{\partial t}$ in equation (29) by the smoothed function $J_{\epsilon}^{-1} \frac{\partial u}{\partial t}$, and using the relation 


$$
A(t) J_{\epsilon}^{-1}=J_{\epsilon}^{-1} A(\tau)+\epsilon J_{\epsilon}^{-1} \frac{\partial A(\tau)}{\partial \tau} J_{\epsilon}^{-1}
$$

we get

$$
\int_{Q} \frac{\partial u}{\partial t} \overline{N \frac{\partial v_{\epsilon}^{*}}{\partial t}} d x d t=\int_{Q} A(t) \frac{\partial u}{\partial t} \overline{v_{\epsilon}^{*}} d x d t+\epsilon \int_{Q} \frac{\partial A}{\partial t}\left(\frac{\partial u}{\partial t}\right)_{\epsilon} \overline{v_{\epsilon}^{*}} d x d t
$$

By passing to the limit, we satisfy the equality (30) for all functions satisfying the conditions in equations (2)-(5), such that $\frac{\partial^{i}}{\partial x^{i}}\left(a \frac{\partial^{\alpha+1} u}{\partial x^{\alpha} \partial t}\right) \in L_{2}(Q)$ for $0 \leq i \leq \alpha$.

The operator $A(t)$ has, on $L_{2}(0,1)$, a continuous inverse defined by the relation

$$
\begin{gathered}
A^{-1}(t) g=(-1)^{\alpha} \int_{0}^{x} \int_{0}^{\eta_{\alpha-1}} \ldots \int_{0}^{\eta_{1}} \frac{1}{a(\eta, t)(1-\eta)}\left[\int_{0}^{\eta} \int_{0}^{\xi_{\alpha-1}} \ldots \int_{0}^{\xi_{1}} g(\xi) d \xi d \xi_{1} \ldots d \xi_{\alpha-1}\right. \\
\left.+\sum_{i=1}^{\alpha} C_{i} \frac{\eta^{i-1}}{(i-1) !}\right] d \eta d \eta_{1} \ldots d \eta_{\alpha-1},
\end{gathered}
$$

where

$$
\int_{0}^{1} A^{-1}(t) g d x=0
$$

Hence the function $\left(\frac{\partial u}{\partial t}\right)_{\epsilon}$ can be represented in the form $\left(\frac{\partial u}{\partial t}\right)_{\epsilon}=J_{\epsilon}^{-1} A^{-1} A \frac{\partial u}{\partial t}$. Then, $\frac{\partial A}{\partial t}\left(\frac{\partial u}{\partial t}\right)_{\epsilon}=A_{\epsilon}(t) A(t) \frac{\partial u}{\partial t}$, where

$$
\begin{gathered}
A_{\epsilon}(t) g=\sum_{k=0}^{\alpha} \mathcal{C}_{\alpha}^{k} \frac{\partial^{k+1} a}{\partial x^{k} \partial t}\left[\sum_{p=0}^{\alpha-k} \frac{\partial^{p}\left(\frac{1}{a}\right)}{\partial x^{p}} \frac{\partial^{\alpha-k-p}}{\partial x^{\alpha-k-p}}\left(\int_{0}^{x} \int_{0}^{\xi_{\alpha-1}} \ldots \int_{0}^{\xi_{1}} g(\xi, t) d \xi d \xi_{1} \ldots d \xi_{\alpha-1}\right)\right]_{\epsilon} \\
+\left[\sum_{k=1}^{\alpha} C_{k} \sum_{\ell=0}^{\alpha}\left\{\mathcal{C}_{\alpha}^{\ell} \frac{\partial^{\ell+1} a}{\partial x^{\ell} \partial t} \sum_{p=0}^{\alpha-\ell}\left(\frac{\partial^{p}\left(\frac{1}{a}\right)}{\partial x^{p}} \frac{\partial^{\alpha-\ell-p}}{\partial x^{\alpha-\ell-p}} \frac{x^{k-1}}{(k-1) !}\right)_{\epsilon}\right\}\right] .
\end{gathered}
$$

Consequently, equation (30) becomes

$$
\int_{Q} \frac{\partial u}{\partial t} \overline{N \frac{\partial v_{\epsilon}^{*}}{\partial t}} d x d t=\int_{Q} A(t) \frac{\partial u}{\partial t}\left(v_{\epsilon}^{*}+\epsilon A_{\epsilon}^{*} v_{\epsilon}^{*}\right) d x d t
$$

in which $A_{\epsilon}^{*}(t)$ is the adjoint of the operator $A_{\epsilon}(t)$.

The left-hand side of equation (34) is a continuous linear functional of $\frac{\partial u}{\partial t}$. Hence the function $h_{\epsilon}=v_{\epsilon}^{*}+\epsilon A_{\epsilon}^{*} v_{\epsilon}^{*}$ has the derivatives $\frac{\partial^{i} h_{\epsilon}}{\partial x^{i}} \in L_{2}(Q), \frac{\partial^{i}}{\partial x^{i}}\left(a(x, t) \frac{\partial^{\alpha} h_{\epsilon}}{\partial x^{\alpha}}\right) \in L_{2}(Q)$, $i=\overline{1, \alpha}$, and the following conditions are satisfied

$$
\left.\frac{\partial^{i} h_{\epsilon}}{\partial x^{i}}\right|_{x=0}=0, i=\overline{0, \alpha-1},\left.\frac{\partial^{i} h_{\epsilon}}{\partial x^{i}}\right|_{x=1}=0, i=\overline{1, \alpha}
$$


The operators $A_{\epsilon}^{*}(t)$ are bounded in $L_{2}(Q)$. For $\epsilon$ sufficiently small, we have $\left\|\epsilon A_{\epsilon}^{*}(t)\right\|_{L_{2}(Q)}<$ 1 , hence the operator, $I+\epsilon A_{\epsilon}^{*}(t)$ has a bounded inverse in $L_{2}(Q)$. In addition, $\frac{\partial^{i} A_{\epsilon}^{*}(t)}{\partial x^{i}}, i=$ $\overline{1, \alpha}$, are bounded operators in $L_{2}(Q)$. So from the equality,

$$
\frac{\partial^{i} h_{\epsilon}}{\partial x^{i}}=\left(I+\epsilon A_{\epsilon}^{*}(t)\right) \frac{\partial^{i} v_{\epsilon}^{*}}{\partial x^{i}}+\epsilon \sum_{k=1}^{i} \mathcal{C}_{i}^{k} \frac{\partial^{k} A_{\epsilon}^{*}(t)}{\partial x^{k}} \frac{\partial^{i-k} v_{\epsilon}^{*}}{\partial x_{i-k}}, \quad i=\overline{0, \alpha}
$$

We conclude that the function $v_{\epsilon}^{*}$ has the derivatives $\frac{\partial^{i} v_{\epsilon}^{*}}{\partial x^{i}} \in L_{2}(Q), i=\overline{0, \alpha}$. Taking into account equations (36) and (35), we have

$$
\left[\left(I+\epsilon A_{\epsilon}^{*}(t)\right) \frac{\partial^{i} v_{\epsilon}^{*}}{\partial x^{i}}+\epsilon \sum_{k=1}^{i} \mathcal{C}_{i}^{k} \frac{\partial^{k} A_{\epsilon}^{*}(t)}{\partial x^{k}} \frac{\partial^{i-k} v_{\epsilon}^{*}}{\partial x^{i-k}}\right]_{x=0}=0, i=\overline{0, \alpha-1}
$$

and

$$
\left[\left(I+\epsilon A_{\epsilon}^{*}(t)\right) \frac{\partial^{i} v_{\epsilon}^{*}}{\partial x^{i}}+\epsilon \sum_{k=1}^{i} \mathcal{C}_{i}^{k} \frac{\partial^{k} A_{\epsilon}^{*}(t)}{\partial x^{k}} \frac{\partial^{i-k} v_{\epsilon}^{*}}{\partial x^{i-k}}\right]_{x=0}=0, i=\overline{0, \alpha} .
$$

Similarly, for $\epsilon$ sufficiently small, in each fixed point $x \in[0,1]$, the operators $\frac{\partial^{i} A_{\epsilon}^{*}(t)}{\partial x^{i}}, i=\overline{0, \alpha}$ are bounded in $L_{2}(Q)$, and the operator $I+\epsilon A_{\epsilon}^{*}(t)$ is continuous inversible in $L_{2}(Q)$. And so from equations (37) and (38), $v_{\epsilon}^{*}$ satisfies the conditions

$$
\begin{gathered}
\left.\frac{\partial^{i} v_{\epsilon}^{*}}{\partial x^{i}}\right|_{x=0}=0, \quad i=\overline{0, \alpha-1}, \\
\left.\frac{\partial^{i} v_{\epsilon}^{*}}{\partial x^{i}}\right|_{x=0}=0, \quad i=\overline{0, \alpha} .
\end{gathered}
$$

So, for $\epsilon$ sufficiently small, the function $v_{\epsilon}^{*}$ has the same properties as $h_{\epsilon}$. In addition, $v_{\epsilon}^{*}$ satisfies the integral condition in equation (28).

Putting $u=\int_{0}^{t} \int_{0}^{\tau} \exp (c \eta) v_{\epsilon}^{*}(\eta, \tau) d \eta d \tau$ in equation (29), where the constant $c$ satisfies $c a_{0}-a_{3}-\frac{a_{3}^{2}}{a_{0}} \geq 0$ and using equation (27), we obtain

$$
\int_{Q} \exp (c t) v_{\epsilon}^{*} \overline{N v} d x d t=-\int_{Q} A(t) \frac{\partial u}{\partial t} \exp (-c t) \frac{\partial^{2} \bar{u}}{\partial t^{2}} d x d t+\epsilon \int_{Q} A(t) \frac{\partial u}{\partial t} \frac{\partial \overline{v_{t}^{*}}}{\partial t} d x d t
$$

Integrating each term in the left-hand side of equation (40) by parts and taking the real parts, we have

$$
\begin{gathered}
\operatorname{Re} \int_{Q} A(t) \frac{\partial u}{\partial t} \exp (-c t) \frac{\partial^{2} \bar{u}}{\partial t^{2}} d x d t \geq \frac{c}{2} \int_{Q} a(x, t) \exp (-c t)(1-x)\left|\frac{\partial^{\alpha+1} u}{\partial x^{\alpha} \partial t}\right|^{2} d x d t \\
-\frac{1}{2} \int_{Q} \frac{\partial a}{\partial t} \exp (-c t)(1-x)\left|\frac{\partial^{\alpha+1} u}{\partial x^{\alpha} \partial t}\right|^{2} d x d t . \\
\operatorname{Re}\left(-\epsilon \int_{Q} A(t) \frac{\partial u}{\partial t} \frac{\partial v_{\epsilon}^{*}}{\partial t} d x d t\right) \\
\geq \frac{-\epsilon a_{3}^{2}}{2 a_{0}} \int_{Q} \exp (-c t)(1-x)\left|\frac{\partial^{\alpha+1} u}{\partial x^{\alpha} \partial t}\right|^{2} d x d t .
\end{gathered}
$$


Now, using inequalities (41) and (42) in equation (40) with the choice of $c$ indicated above, we have $2 \operatorname{Re} \int_{Q} \exp (c t) v_{\epsilon}^{*} \overline{N v} d x d t \leq 0$; then for $\epsilon \rightarrow 0$, we obtain $2 \operatorname{Re} \int_{Q} \exp (c t) v \overline{N v} d x d t \leq 0$, i.e., $2 \operatorname{Re} \int_{Q} \exp (c t)(1-x)|v|^{2} d x d t+2 \operatorname{Re} \int_{Q} \exp (c t) v J \bar{v} d x d t \leq 0$.

Since $\operatorname{Re} \int_{Q} \exp (c t) v J \bar{v} d x d t=0$, we conclude that $v=0$; hence $\omega=0$, which ends the proof of this lemma.

Theorem 2: The range $R(\bar{L})$ of $\bar{L}$ coincides with $F$.

Proof: Since $F$ is a Hilbert space, we have $R(\bar{L})=F$ if and only if the relation

$$
\int_{Q}(1-x)^{\nu} \mathcal{L} u \bar{f} d x d t+\int_{0}^{1}(1-x)\left(\sum_{i=0}^{\alpha} \frac{\partial^{i} l_{1} u}{\partial x^{i}} \frac{\partial^{i} \bar{\varphi}}{\partial x^{i}}+\frac{\partial^{\alpha} l_{2} u}{\partial x^{\alpha}} \frac{\partial^{\alpha} \bar{\psi}}{\partial x^{\alpha}}+l_{2} u \bar{\psi}\right) d x=0 .
$$

for arbitrary $u \in E$ and $\mathcal{F}=(f, \varphi, \psi) \in F$, implies that $f=0, \varphi=0$ and $\psi=0$.

Putting $u \in D_{0}(L)$ in relation (43), we obtain $\int_{Q}(1-x)^{\nu} \mathcal{L} u \bar{f} d x d t=0$. Taking $\omega=\frac{f}{(1-x)^{\alpha+1}}$, and using Lemma 5, we obtain $\omega=\frac{f}{(1-x)^{\alpha+1}}=0$, then $f=0$.

Consequently, $\forall u \in D(L)$ we have

$$
\int_{0}^{1}(1-x)\left(\sum_{i=0}^{\alpha} \frac{\partial^{i} l_{1} u}{\partial x^{i}} \frac{\partial^{i} \bar{\varphi}}{\partial x^{i}}+\frac{\partial^{\alpha} l_{2} u}{\partial x^{\alpha}} \frac{\partial^{\alpha} \bar{\psi}}{\partial x^{\alpha}}+l_{2} u \bar{\psi}\right) d x=0
$$

The range of the trace operator $\left(l_{1}, l_{2}\right)$ is everywhere dense in Hilbert space with the norm

$$
\left[\int_{0}^{1}(1-x)\left(\sum_{i=0}^{\alpha}\left|\frac{\partial^{i} \varphi}{\partial x^{i}}\right|^{2}+\left|\frac{\partial^{\alpha} \psi}{\partial x^{\alpha}}\right|^{2}+|\psi|^{2}\right) d x\right]^{\frac{1}{2}}
$$

hence $(\varphi, \psi)=(0,0)$.

\section{References}

[1] Batten, Jr., G.W., Second-order correct boundary conditions for the numerical solution of the mixed boundary problem for parabolic equations, Math. Comp. 17 (1963), 405-413.

[2] Bouziani, A. and Benouar, N.E., Mixed problem with integral conditions for a third order parabolic equation, Kobe J. Math. 15 (1998), 47-58.

[3] Cannon, J.R., The solution of the heat equation subject to the specification of energy, Quart. Appl. Math. 21 (1963), 155-160.

[4] Cannon, J.R., The one-dimensional heat equation, In: Encyclopedia of Math. and its Appl. 23, Addison-Wesley, Mento Park, CA (1984).

[5] Cannon, J.R., Perez Esteva, S., and Van Der Hoek, J., A Galerkin procedure for the diffusion equation subject to the specification of mass, SIAM. J. Numer. Anal. 24 (1987), 499-515.

[6] Choi, Y.S. and Chan, K.Y., A parabolic equation with nonlocal boundary conditions arising from electrochemistry, Nonl. Anal. 18 (1992), 317-331. 
[7] Ewing, R.E. and Lin, T., A class of parameter estimation techniques for fluid flow in porous media, Adv. Water Resources 14 (1991), 89-97.

[8] Ionkin, N.I., Solution of a boundary value problem in heat condition with a nonclassical boundary condition, Diff. Urav. 13 (1977), 294-304.

[9] Kamynin, N.I., A boundary value problem in the theory of the heat condition with nonclassical boundary condition, USSR Comput. Math. and Math. Phys. 4 (1964), 33-59.

[10] Kartynnik, A.V., Three-point boundary value problem with an integral space-variable condition for a second-order parabolic equation, Differ. Eqns. 26 (1990), 1160-1166.

[11] Rivlin, T.J., The Chebyshev Polynomials, John Wiley \& Sons, New York 1974.

[12] Shi, P., Weak solution to evolution problem with a nonlocal constraint, SIAM J. Anal. 24 91993), 46-58.

[13] Shi, P. and Shillor, M., Design of Contact Patterns in One-Dimensional Thermoelasticity, In: Theoretical Aspects of Industrial Design, SIAM, Philadelphia, PA 1992.

[14] Yurchuk, N.I., Mixed problem with an integral condition for certain parabolic equations, Differ. Eqns. 22 (1986), 1457-1463. 


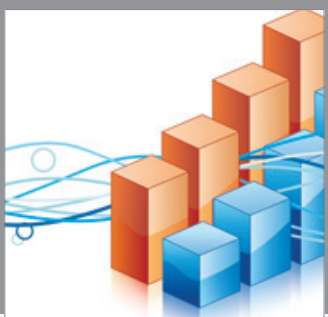

Advances in

Operations Research

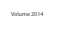

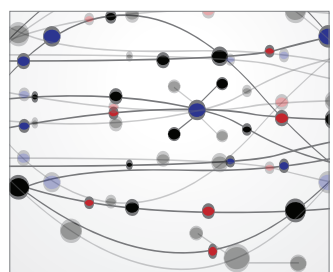

\section{The Scientific} World Journal
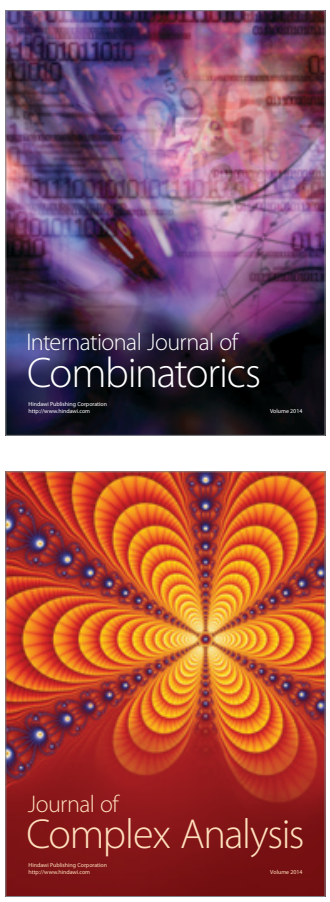

International Journal of

Mathematics and

Mathematical

Sciences
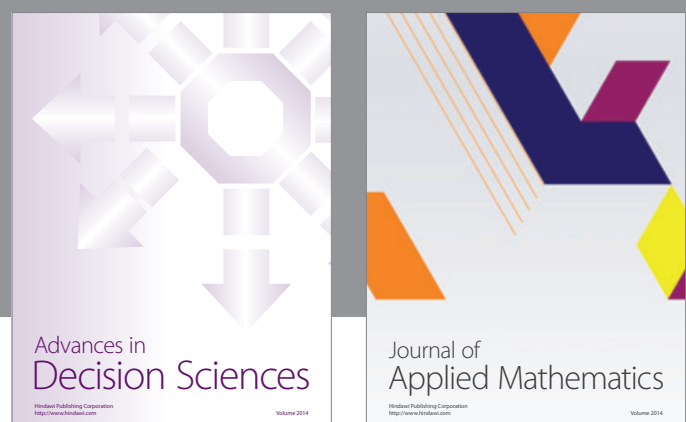

Journal of

Applied Mathematics
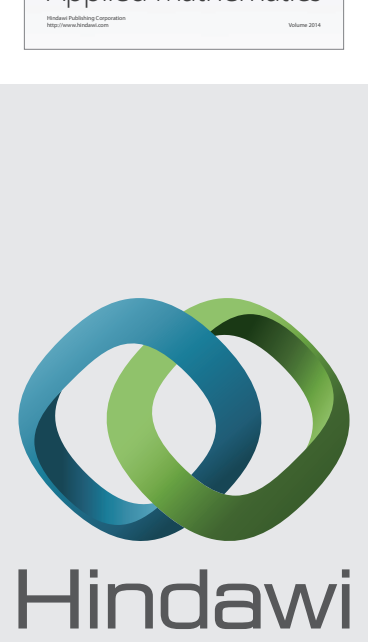

Submit your manuscripts at http://www.hindawi.com
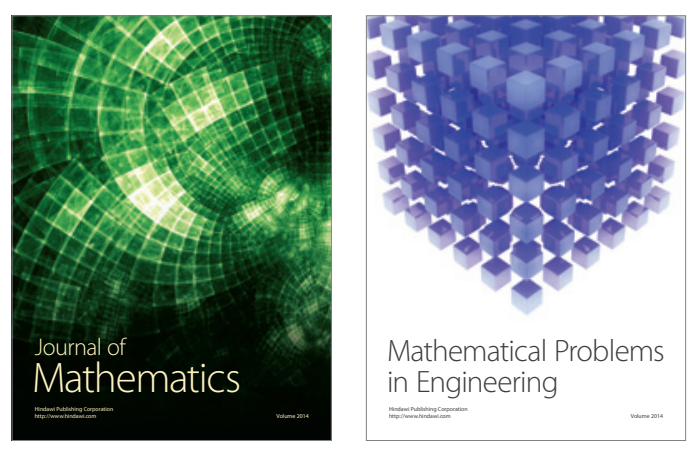

Mathematical Problems in Engineering
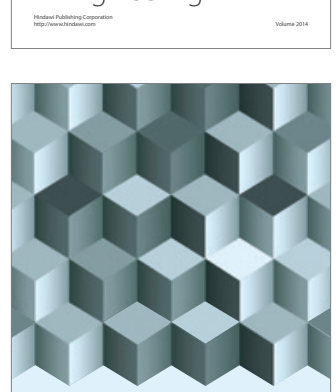

Journal of

Function Spaces
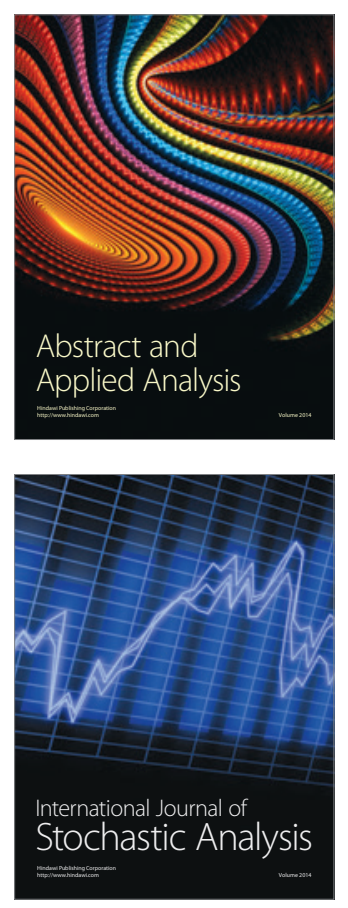

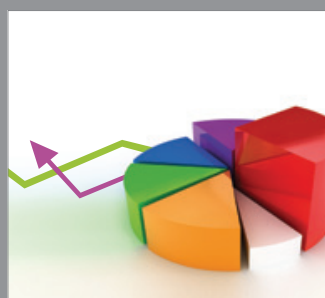

ournal of

Probability and Statistics

Promensencen
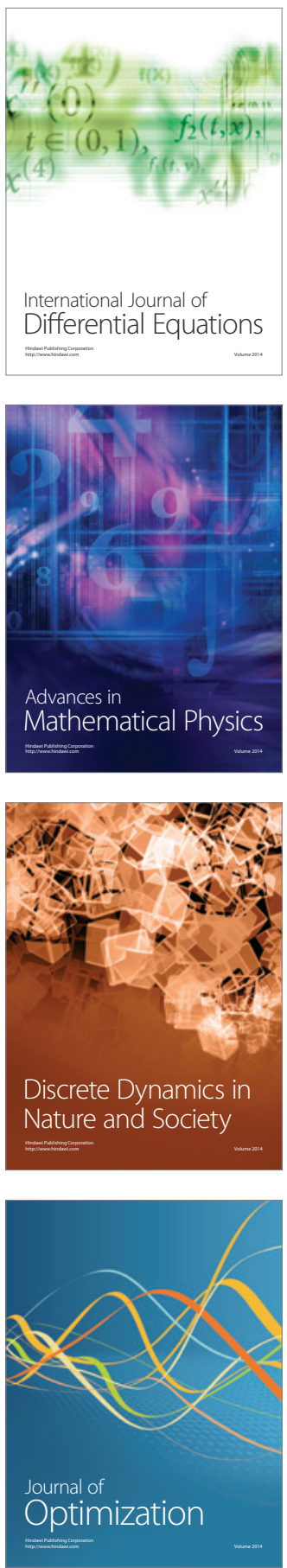